\title{
Computing The Kinematics Study of a 6 DOF Industrial Manipulator Prototype By Matlab
}

\author{
Alaa Saadah \\ Mechatronical Engineering Department \\ The University of Debrecen \\ Debrecen, Hungary \\ alaa.saadah@eng.unideb.hu
}

\author{
Husam Abdulkareem \\ Mechatronical Engineering Department \\ The University of Debrecen \\ Debrecen, Hungary \\ husam@eng.unideb.hu
}

\author{
Géza Husi \\ Mechatronical Engineering Department \\ The University of Debrecen \\ Debrecen, Hungary \\ husigeza@eng.unideb.hu
}

\begin{abstract}
Since the mechanical parts in the robot are designed to do the movement, studying and analyzing the motion considered a primary issue that should be taken into consider when studying and designing the robot.

In this research 6-DOF sample of the industrial manipulator based on ABB IRB 4400 model had been studied. The mathematical model of manipulator is established by DH (Denavait Hartenberg) method. The forward kinematics was done using DH Parameters in order to get the final transformation matrix. The inverse kinematics was done using geometrical and analytical methods in order to get the end effector final position and direction by calculate Euler angles values.
\end{abstract}

Finally, the forward and inverse kinematics equations were computing by MATLAB to get angles, end effector, position, direction and Euler angles values.

The kinematics study and the arm movement's equations were compered with the practical measurements to make sure it fulfills the desired purpose.

Keywords- 6-DOF Industrial manipulator, Forward Kinematics, Inverse Kinematics.

\section{INTRODUCTION}

In this twenty first century, due to the heavy demand for high quality and great accuracy product from the customer. A large number of industries nowadays shifted their focus toward the installation of the robotic arm in their assembly line for faster production of the product.

One of the most challenging problems of the robotic system is kinematics study, specially the inverse kinematics which deals to find the joint angles for the given robotic configurations.

The manipulator of industrial robot consists of a series of joints and links, ABB IRB 4400 manipulator sample deals with the study of different joint and link and other aspect of the manipulator's physical construction.

\section{ANALYZING THE MOVEMENT}

The robotic kinematics is a since specialized in studying each moving part of the robot without studying the case of that's part's movement.

\section{A. Forward Kinematics:}

The forward kinematics problem is concerned with the relationship between the individual joints of the robot manipulator and position and orientation of the tool or endeffector [1].

Stated more formally, the forward kinematics problem is to determine the position and orientation of the end-effector, given the values for the joint variables of the robot. The joint variables are the angles between the links in the case of prismatic or sliding [2].

A robot manipulator with $n$ joint will have $n+1$ link, since each joint connects two links. We number the joint from 1 to $\mathrm{n}$, and we number the link from 0 to $\mathrm{n}$ starting from the base.

By this convention, joint $\mathrm{i}$ connects link i-1 to link i moves. Therefore, link 0 - the first link- is fixed and does not move when the joint is actuated.

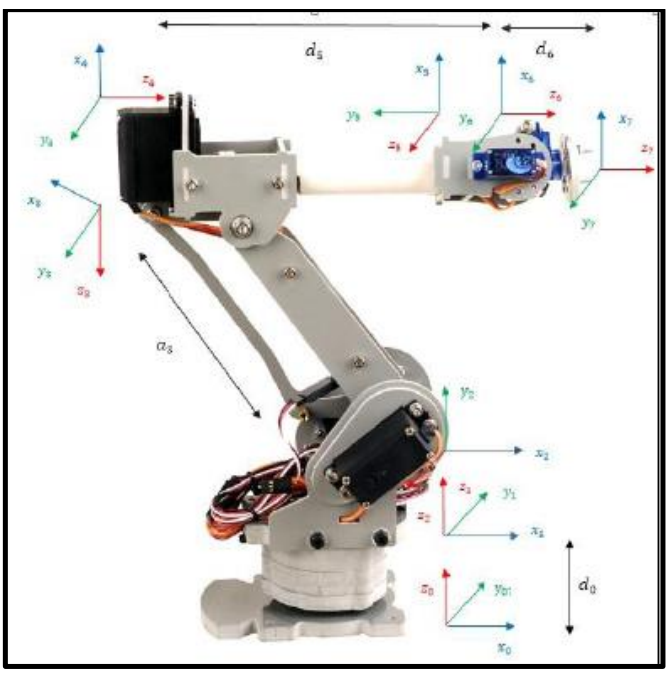

Figure (1): ABB IRB 4400 Sample Frame Assignment [photographed by authors]

\section{B. Denavait Hartenberg:}

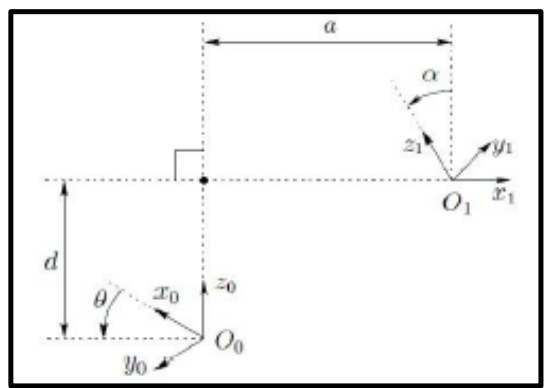

Figure (2): DH Frame Assignment [1]. 
We can get Ti depend on four primary transformation:

$T_{i}=\operatorname{Rot}\left(z, \theta_{i}\right) \operatorname{Trans}\left(z, d_{i}\right) \operatorname{Trans}\left(x, a_{i}\right) \operatorname{Rot}\left(x, \alpha_{i}\right)$

So, we can transfer from i frame to $i+1$ frame

$\operatorname{Rot}\left(x, \alpha_{i}\right)$ : Rotation around $x$ axes $a_{i}$ angle.

$\operatorname{Trans}\left(x, a_{i}\right)$ : Transformation $x$ axes $a_{i}$ angle.

$\operatorname{Rot}\left(z, \theta_{i}\right)$ : Rotation around $z$ axes $\theta_{i}$ angle.

$\operatorname{Trans}\left(z, d_{i}\right)$ : Transformation $z$ axes $d_{i}$ angle.

$T_{i}$ Final matrix will be the result of multiple of those matrixes [3].

$$
\begin{aligned}
& T_{i}= {\left[\begin{array}{llll}
c_{\theta i} & -s_{\theta i} & 0 & 0 \\
s_{\theta i} & c_{\theta i} & 0 & 0 \\
0 & 0 & 1 & 0 \\
0 & 0 & 0 & 1
\end{array}\right] \cdot\left[\begin{array}{cccc}
1 & 0 & 0 & 0 \\
0 & 1 & 0 & 0 \\
0 & 0 & 1 & d_{i} \\
0 & 0 & 0 & 1
\end{array}\right] \cdot\left[\begin{array}{cccc}
1 & 0 & 0 & a_{i} \\
0 & 1 & 0 & 0 \\
0 & 0 & 1 & 0 \\
0 & 0 & 0 & 1
\end{array}\right] \cdot\left[\begin{array}{cccc}
1 & 0 & 0 & 0 \\
0 & c_{\alpha i} & -s_{\alpha i} & 0 \\
0 & s_{\alpha i} & c_{\alpha i} & 0 \\
0 & 0 & 0 & 1
\end{array}\right] } \\
& T_{i}=\left[\begin{array}{cccc}
c_{\theta i} & -s_{\theta i} c_{\alpha i} & s_{\theta i} s_{\alpha i} & a_{i} c_{\theta i} \\
s_{\theta i} & c_{\theta i} c_{\alpha i} & -c_{\theta i} s_{\alpha i} & a_{i} s_{\alpha i} \\
0 & s_{\alpha i} & c_{\alpha i} & d_{i} \\
0 & 0 & 0 & 1
\end{array}\right] \\
& \alpha_{i}, \theta_{i}, d_{i}, a_{i}
\end{aligned}
$$

$a_{i}$ : The distance between $z_{i}$ to $z_{i+1}$ along the $x_{i}$ axis.

$\alpha_{i}$ : The angle between $z_{i}$ to $z_{i+1}$ about $x_{i}$ axis.

$d_{i}$ : The distance between $x_{i}$ to $x_{i+1}$ along the $z_{i}$ axis.

$\theta_{i}$ : The angle between $x_{i}$ to $x_{i+1}$ about $z_{i}$ axis.

\section{Homogeneous transformation}

$H=\operatorname{Rot}_{x, \alpha} \operatorname{Trans}_{x, a} \operatorname{Trans}_{z, d} \operatorname{Rot}_{z, \theta}$

We get the homogeneous transformation $\mathrm{H}$ by rotate $\alpha$ angle about $\alpha$ axis then translation among $x$ axis then translation among $z$ axis and finally rotate $\theta$ angle about $z$ axis [4].

$H=\left[\begin{array}{cccc}c_{\theta} & -s_{\theta} & 0 & a \\ c_{\alpha} s_{\theta} & c_{\theta} c_{\alpha} & -s_{\alpha} & -d s_{\alpha} \\ s_{\alpha} s_{\theta} & s_{\alpha} c_{\theta} & c_{\alpha} & d c_{\alpha} \\ 0 & 0 & 0 & 1\end{array}\right]$

The homogeneous that given by this matrix is a special status and to generalize it we should take into consider that Scale Factor $=1$ and Perspective Transformation $=\left[\begin{array}{lll}0 & 0 & 0\end{array}\right]$.

So, the final matrix will be

$H=\left[\begin{array}{ll}R_{3 \times 3} & d_{3 \times 1} \\ f_{1 \times 3} & S_{1 \times 1}\end{array}\right]=\left[\begin{array}{cc}\text { Rotation } & \text { Translation } \\ \text { Perspective } & \text { Scale factor }\end{array}\right]$

$H=\left[\begin{array}{cccc}\mu_{x} & O_{x} & \alpha_{x} & p_{x} \\ \mu_{y} & O_{y} & \alpha_{y} & p_{y} \\ \mu_{z} & O_{z} & \alpha_{z} & p_{z} \\ 0 & 0 & 0 & 1\end{array}\right]$

Then, forward kinematics given by multiply all transforming matrixes together

$$
T_{i}^{j}=T_{j+1}^{j} \cdots \cdots T_{i}^{i-1}
$$

We will use homogeneous transformation $T_{i}^{i-1}$ which transfer us from i frame to i-1 frame.

$$
T_{i}^{i-1}=T_{i}^{i-1}\left(q_{i}\right)
$$

Depending on previous equation, the matrix $T_{i}^{j}(i>j)$

Will transfer us from $i$ frame to $j$ frame given by

$T_{n}^{0}=\left[\begin{array}{cc}R_{n}^{0} & d_{n}^{0} \\ 0 & 1\end{array}\right]$

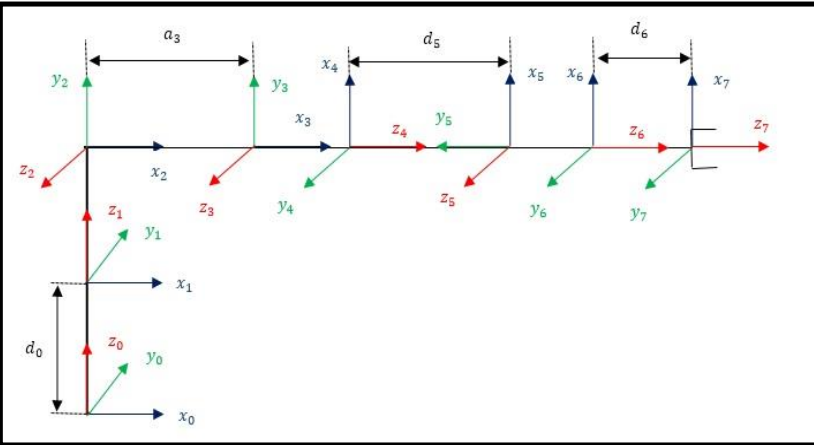

Hence, the position and direction for the end-effector given by

$$
\begin{aligned}
& T_{n}^{0}\left(q_{1}, q_{2}, \ldots, q_{n}\right)=T_{1}^{0}\left(q_{1}\right) \cdot T_{2}^{1}\left(q_{2}\right) \ldots . T_{n}^{n-1}\left(q_{n}\right) \\
& T_{i}^{j}=T_{j+1}^{j} \ldots T_{i}^{i-1}=\left[\begin{array}{cc}
R_{i}^{j} & d_{i}^{j} \\
0 & 1
\end{array}\right]
\end{aligned}
$$

Figure (3): Coordinate Frames of ABB IRB 4400 Manipulator Sample [Created by authors]

\section{DH Parameters}

Table (1): Denavait Hartenberg Parameters

\begin{tabular}{|c|c|c|c|c|}
\hline $\boldsymbol{i}$ & $\boldsymbol{\theta}_{\boldsymbol{i}-\mathbf{1}}$ & $\boldsymbol{d}_{\boldsymbol{i}-\mathbf{1}}$ & $\boldsymbol{a}_{\boldsymbol{i}}$ & $\boldsymbol{\alpha}_{\boldsymbol{i}}$ \\
\hline 1 & 0 & $d_{0}$ & 0 & 0 \\
\hline 2 & $q_{1}$ & 0 & 0 & $90^{\circ}$ \\
\hline 3 & $q_{2}$ & 0 & $a_{3}$ & 0 \\
\hline 4 & $90^{\circ}+q_{3}$ & 0 & 0 & $90^{\circ}$ \\
\hline 5 & $q_{4}$ & $d_{4}$ & 0 & $-90^{\circ}$ \\
\hline 6 & $q_{5}$ & 0 & 0 & $90^{\circ}$ \\
\hline 7 & $q_{6}$ & $d_{6}$ & 0 & 0 \\
\hline
\end{tabular}

$$
\begin{aligned}
T_{1}^{0} & =\left[\begin{array}{llll}
1 & 0 & 0 & 0 \\
0 & 1 & 0 & 0 \\
0 & 0 & 1 & d_{0} \\
0 & 0 & 0 & 1
\end{array}\right] \\
T_{2}^{1} & =\left[\begin{array}{cccc}
c_{q_{1}} & 0 & s_{q_{1}} & 0 \\
s_{q_{1}} & 0 & -c_{q_{1}} & 0 \\
0 & 1 & 0 & 0 \\
0 & 0 & 0 & 1
\end{array}\right] \\
T_{3}^{2} & =\left[\begin{array}{cccc}
c_{q_{2}} & -s_{q_{2}} & 0 & c_{q_{2}} a_{3} \\
s_{q_{2}} & c_{q_{2}} & 0 & s_{q_{2}} a_{3} \\
0 & 0 & 1 & 0 \\
0 & 0 & 0 & 1
\end{array}\right]
\end{aligned}
$$

$T_{4}^{3}=\left[\begin{array}{cccc}-s_{q_{3}} & 0 & c_{q_{3}} & 0 \\ c_{q_{3}} & 0 & s_{q_{3}} & 0 \\ 0 & 1 & 0 & 0 \\ 0 & 0 & 0 & 1\end{array}\right]$

$T_{5}^{4}=\left[\begin{array}{cccc}c_{q_{4}} & 0 & -s_{q_{4}} & 0 \\ s_{q_{4}} & 0 & c_{q_{4}} & 0 \\ 0 & -1 & 0 & d_{5} \\ 0 & 0 & 0 & 1\end{array}\right]$ 


$$
\begin{aligned}
& T_{6}^{5}=\left[\begin{array}{cccc}
c_{q_{5}} & 0 & s_{q_{5}} & 0 \\
s_{q_{5}} & 0 & -c_{q_{5}} & 0 \\
0 & 1 & 0 & 0 \\
0 & 0 & 0 & 1
\end{array}\right] \\
& T_{7}^{6}=\left[\begin{array}{cccc}
c_{q_{6}} & -s_{q_{6}} & 0 & 0 \\
s_{q_{6}} & c_{q_{6}} & 0 & 0 \\
0 & 0 & 1 & d_{6} \\
0 & 0 & 0 & 1
\end{array}\right]
\end{aligned}
$$

$$
T_{7}^{0}=T_{1}^{0} \cdot T_{2}^{1} \cdot T_{3}^{2} \cdot T_{4}^{3} \cdot T_{5}^{4} \cdot T_{6}^{5} \cdot T_{7}^{6}=\left[\begin{array}{cccc}
\mu_{x} & O_{x} & \alpha_{x} & p_{x} \\
\mu_{y} & O_{y} & \alpha_{y} & p_{y} \\
\mu_{z} & O_{z} & \alpha_{z} & p_{z} \\
0 & 0 & 0 & 1
\end{array}\right](13)
$$$$
\mu_{x}=-c_{q_{1}} c_{q_{2}} s_{q_{3}} c_{q_{4}} c_{q_{5}} c_{q_{6}}+c_{q_{1}} c_{q_{2}} s_{q_{3}} s_{q_{4}} s_{q_{6}}-
$$$$
c_{q_{1}} c_{q_{2}} c_{q_{3}} s_{q_{5}} c_{q_{6}}-c_{q_{1}} s_{q_{2}} c_{q_{3}} c_{q_{4}} c_{q_{5}} c_{q_{6}}+
$$$$
c_{q_{1}} s_{q_{2}} c_{q_{3}} s_{q_{4}} s_{q_{6}}+c_{q_{1}} s_{q_{2}} s_{q_{3}} s_{q_{5}} c_{q_{6}}+s_{q_{1}} s_{q_{4}} c_{q_{5}} c_{q_{6}}+
$$$$
s_{q_{1}} c_{q_{4}} s_{q_{6}}
$$$$
\mu_{y}=-s_{q_{1}} c_{q_{2}} s_{q_{3}} c_{q_{4}} c_{q_{5}} c_{q_{6}}+s_{q_{1}} c_{q_{2}} s_{q_{3}} s_{q_{4}} s_{q_{6}}-
$$$$
s_{q_{1}} c_{q_{2}} c_{q_{3}} s_{q_{5}} c_{q_{6}}-s_{q_{1}} s_{q_{2}} c_{q_{3}} c_{q_{4}} c_{q_{5}} c_{q_{6}}+
$$$$
s_{q_{1}} s_{q_{2}} c_{q_{3}} s_{q_{4}} s_{q_{6}}+s_{q_{1}} s_{q_{2}} s_{q_{3}} s_{q_{5}} c_{q_{6}}-c_{q_{1}} s_{q_{4}} c_{q_{5}} c_{q_{6}}-
$$$$
c_{q_{1}} c_{q_{4}} s_{q_{6}}
$$$$
\mu_{z}=-s_{q_{2}} s_{q_{3}} c_{q_{4}} c_{q_{5}} c_{q_{6}}+s_{q_{2}} s_{q_{3}} s_{q_{4}} s_{q_{6}}-
$$$$
s_{q_{2}} c_{q_{3}} s_{q_{5}} c_{q_{6}}+c_{q_{2}} c_{q_{3}} c_{q_{4}} c_{q_{5}} c_{q_{6}}-c_{q_{2}} c_{q_{3}} s_{q_{4}} s_{q_{6}}-
$$$$
c_{q_{2}} s_{q_{3}} s_{q_{5}} c_{q_{6}}
$$

$$
O_{x}=c_{q_{1}} c_{q_{2}} s_{q_{3}} c_{q_{5}} s_{q_{6}}+c_{q_{1}} c_{q_{2}} s_{q_{3}} s_{q_{4}} s_{q_{6}}+
$$$$
c_{q_{1}} c_{q_{2}} c_{q_{3}} s_{q_{5}} s_{q_{6}}+c_{q_{1}} s_{q_{2}} c_{q_{3}} c_{q_{5}} s_{q_{6}}
$$$$
+c_{q_{1}} s_{q_{2}} c_{q_{3}} s_{q_{4}} s_{q_{6}}-c_{q_{1}} s_{q_{2}} s_{q_{3}} s_{q_{5}} s_{q_{6}}-s_{q_{1}} s_{q_{4}} c_{q_{5}} s_{q_{6}}
$$$$
+s_{q_{1}} c_{q_{4}} c_{q_{6}}
$$

$$
O_{y}=s_{q_{1}} c_{q_{2}} s_{q_{3}} c_{q_{5}} s_{q_{6}}+s_{q_{1}} c_{q_{2}} s_{q_{3}} s_{q_{4}} s_{q_{6}}+
$$$$
s_{q_{1}} c_{q_{2}} c_{q_{3}} s_{q_{5}} s_{q_{6}}+s_{q_{1}} s_{q_{2}} c_{q_{3}} c_{q_{5}} s_{q_{6}}+
$$$$
s_{q_{1}} s_{q_{2}} c_{q_{3}} s_{q_{4}} s_{q_{6}}-s_{q_{1}} s_{q_{2}} s_{q_{3}} s_{q_{5}} s_{q_{6}}+c_{q_{1}} s_{q_{4}} c_{q_{5}} s_{q_{6}}-
$$$$
c_{q_{1}} c_{q_{4}} c_{q_{6}}
$$$$
O_{z}=s_{q_{2}} s_{q_{3}} c_{q_{5}} s_{q_{6}}+s_{q_{2}} s_{q_{3}} s_{q_{4}} s_{q_{6}}+s_{q_{2}} c_{q_{3}} s_{q_{5}} s_{q_{6}}-
$$$$
c_{q_{2}} c_{q_{3}} c_{q_{5}} s_{q_{6}}-c_{q_{2}} c_{q_{3}} s_{q_{4}} s_{q_{6}}+c_{q_{2}} s_{q_{3}} s_{q_{5}} s_{q_{6}}
$$$$
a_{x}=-c_{q_{1}} c_{q_{2}} s_{q_{3}} c_{q_{4}} s_{q_{5}}+c_{q_{1}} c_{q_{2}} c_{q_{3}} c_{q_{5}}-
$$$$
c_{q_{1}} s_{q_{2}} c_{q_{3}} c_{q_{4}} s_{q_{5}}-c_{q_{1}} s_{q_{2}} s_{q_{3}} c_{q_{5}}+s_{q_{1}} s_{q_{4}} s_{q_{5}}
$$$$
a_{y}=-s_{q_{1}} c_{q_{2}} s_{q_{3}} c_{q_{4}} s_{q_{5}}+s_{q_{1}} c_{q_{2}} c_{q_{3}} c_{q_{5}}-
$$$$
s_{q_{1}} s_{q_{2}} c_{q_{3}} c_{q_{4}} s_{q_{5}}-s_{q_{1}} s_{q_{2}} s_{q_{3}} c_{q_{5}}-c_{q_{1}} s_{q_{4}} s_{q_{5}}
$$$$
a_{z}=-s_{q_{2}} s_{q_{3}} c_{q_{4}} s_{q_{5}}+s_{q_{2}} c_{q_{3}} c_{q_{5}}+c_{q_{2}} c_{q_{3}} c_{q_{4}} s_{q_{5}}+
$$$$
c_{q_{2}} s_{q_{3}} c_{q_{5}}
$$$$
p_{x}=-c_{q_{1}} c_{q_{2}} s_{q_{3}} c_{q_{4}} s_{q_{5}} d_{6}+c_{q_{1}} c_{q_{2}} c_{q_{3}} c_{q_{5}} d_{6}+
$$$$
c_{q_{1}} c_{q_{2}} c_{q_{3}} d_{4}-c_{q_{1}} s_{q_{2}} c_{q_{3}} c_{q_{4}} s_{q_{5}} d_{6}-c_{q_{1}} s_{q_{2}} s_{q_{3}} c_{q_{5}} d_{6}-
$$$$
c_{q_{1}} s_{q_{2}} s_{q_{3}} d_{4}+c_{q_{1}} c_{q_{2}} a_{3}+s_{q_{1}} s_{q_{4}} s_{q_{5}} d_{6}
$$

$$
\begin{gathered}
p_{y}=-s_{q_{1}} c_{q_{2}} s_{q_{3}} c_{q_{4}} s_{q_{5}} d_{6}+s_{q_{1}} c_{q_{2}} c_{q_{3}} c_{q_{5}} d_{6}+ \\
s_{q_{1}} c_{q_{2}} c_{q_{3}} d_{4}-s_{q_{1}} s_{q_{2}} c_{q_{3}} c_{q_{4}} s_{q_{5}} d_{6}-s_{q_{1}} s_{q_{2}} s_{q_{3}} c_{q_{5}} d_{6}- \\
s_{q_{1}} s_{q_{2}} s_{q_{3}} d_{4}+s_{q_{1}} c_{q_{2}} a_{3}-c_{q_{1}} s_{q_{4}} s_{q_{5}} d_{6} \\
p_{z}=-s_{q_{2}} s_{q_{3}} c_{q_{4}} s_{q_{5}} d_{6}+s_{q_{2}} c_{q_{3}} c_{q_{5}} d_{6}+s_{q_{2}} c_{q_{3}} d_{4}+ \\
c_{q_{2}} c_{q_{3}} c_{q_{4}} s_{q_{5}} d_{6}+c_{q_{2}} s_{q_{3}} c_{q_{5}} d_{6}+c_{q_{2}} s_{q_{3}} d_{4}+s_{q_{2}} a_{3}+ \\
d_{0}
\end{gathered}
$$

\section{E. Inverse Kinematics:}

Inverse Kinematics (IK) analysis determines the joint angles for desired position and orientation in Cartesian space. Total transformation matrix in equation. (13) will be used to calculate inverse kinematics equations. IK is more difficult problem than forward kinematics [5].

The solution of inverse kinematic is more complex than direct kinematics and there is not any global analytical solution method. Each manipulator needs a particular method considering the system structure and restrictions. There are

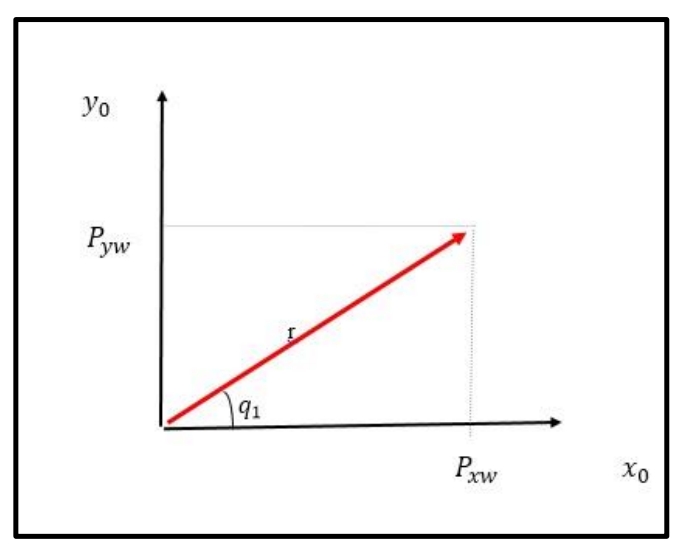

solutions approaches namely, geometric and algebraic used for deriving the inverse kinematics solution. Let's start with geometric approach [6].

\section{- Geometric Approach}

From figure (4) we clearly see that we can calculate q1 as following.

Figure (4): Top View of Robot Arm [Created by authors].

$$
\begin{aligned}
& q_{1}=\tan ^{-1}\left(\frac{p_{y w}}{p_{x w}}\right)=a \tan 2\left(p_{y w}, p_{x w}\right) \\
& q_{1}=\pi+\tan ^{-1}\left(\frac{p_{y w}}{p_{x w}}\right)=a \tan 2\left(-p_{y w},-p_{x w}\right)
\end{aligned}
$$


Moving now to the planar view in Figure (3.4), we find q2, q3 as follows:

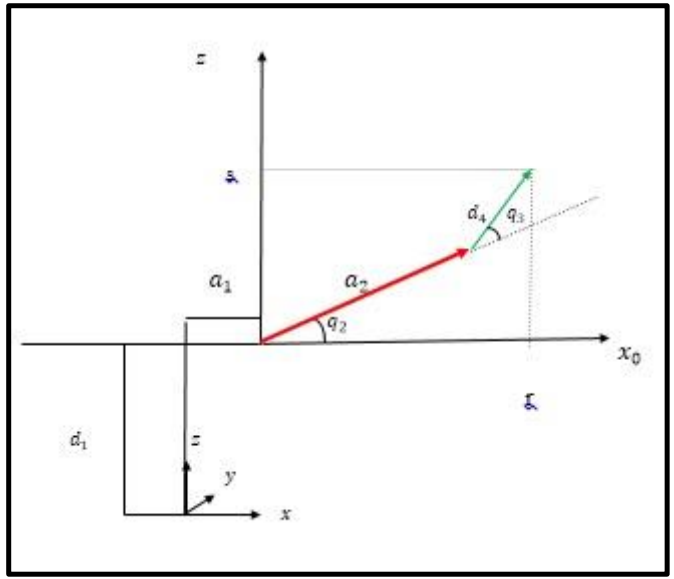

Figure (5): Planar View of Robot Arm [Created by authors].

$$
\begin{gathered}
\cos q_{3}=\frac{\left(p_{x w}+a_{1} \cos \left(q_{1}\right)\right)^{2}+\left(p_{y w}+a_{1} \sin \left(q_{1}\right)\right)^{2}+\left(p_{z w}-d_{1}\right)^{2}-a_{2}{ }^{2}-d_{4}{ }^{2}}{2 a_{2} d_{4}} \\
\cos q_{3}=D \\
q_{3}=\operatorname{acos}(D)
\end{gathered}
$$

There are two values for $\mathrm{q} 3$, which we need to study two cases: Elbow Up and Elbow Down [7].

$$
\begin{gathered}
q_{3}=\operatorname{atan}\left(\frac{ \pm \sqrt{1-D^{2}}}{D}\right) \\
q_{2}=\operatorname{atan}\left(p_{z w}-\frac{d_{1}}{\sqrt{\left(p_{x w}+a_{1} \cos \left(q_{1}\right)\right)^{2}+\left(p_{y w}+a_{1} \sin \left(q_{1}\right)\right)^{2}}}\right. \\
\operatorname{atan}\left(\frac{d_{4} \sin \left(q_{3}\right)}{a_{2}+d_{4} \cos \left(q_{3}\right)}\right)
\end{gathered}
$$

\section{- Analytical (algebraic) Approach}

Using the matrix, we got in direct kinematics [8].

$$
\begin{gathered}
T_{0}^{7}=\left[\begin{array}{cccc}
\mu_{x} & O_{x} & \alpha_{x} & p_{x} \\
\mu_{y} & O_{y} & \alpha_{y} & p_{y} \\
\mu_{z} & O_{z} & \alpha_{z} & p_{z} \\
0 & 0 & 0 & 1
\end{array}\right]=\left[\begin{array}{cccc}
R_{11} & R_{12} & R_{13} & p_{x} \\
R_{21} & R_{22} & R_{23} & p_{y} \\
R_{31} & R_{32} & R_{33} & p_{z} \\
0 & 0 & 0 & 1
\end{array}\right] \\
c\left(q_{4}\right) \cdot s\left(q_{5}\right)=\frac{1}{2}\left[\left(c\left(90-q_{1}+q_{2}+q_{3}\right)+s\left(q_{1}+q_{2}+\right.\right.\right.
\end{gathered}
$$

$\left.\left.q_{3}\right)\right) R_{13}+\left(c\left(q_{1}+q_{2}+q_{3}\right)-s\left(90-q_{1}+q_{2}+q_{3}\right)\right) R_{23}+$ $\left.\left(2 c\left(q_{2}+q_{3}\right)\right) R_{33}\right]$

$$
\begin{gathered}
s\left(q_{4}\right) \cdot s\left(q_{5}\right)=\left[\left(s\left(q_{1}\right)\right) R_{13}-\left(c\left(q_{1}\right)\right) R_{23}\right] \\
q_{4}=\operatorname{atan}\left(\frac{s\left(q_{4}\right) \cdot s\left(q_{5}\right)}{c\left(q_{4}\right) \cdot s\left(q_{5}\right)}\right) \\
s\left(q_{5}\right)=\left[\left(-c\left(q_{1}\right) \cdot c\left(q_{4}\right) \cdot s\left(q_{2}+q_{3}\right)+s\left(q_{1}\right) \cdot s\left(q_{4}\right)\right) R_{13}-\right. \\
\left(c\left(q_{3}\right) \cdot c\left(q_{4}\right) \cdot s\left(q_{1}\right) \cdot s\left(q_{2}\right)+c\left(q_{2}\right) \cdot c\left(q_{4}\right) \cdot s\left(q_{1}\right) \cdot s\left(q_{3}\right)+\right. \\
\left.\left.c\left(q_{1}\right) \cdot s\left(q_{4}\right)\right) R_{23}+\left(c\left(q_{2}+q_{3}\right) \cdot c\left(q_{4}\right)\right) R_{33}\right]
\end{gathered}
$$

$c\left(q_{5}\right)=\frac{1}{2}\left[\left(c\left(q_{1}+q_{2}+q_{3}\right)+s\left(90-q_{1}+q_{2}+q_{3}\right)\right) R_{13}+\right.$ $\left(c\left(90-q_{1}+q_{2}+q_{3}\right)+s\left(q_{1}+q_{2}+q_{3}\right)\right) R_{23}+\left(2 s\left(q_{2}+\right.\right.$ $\left.\left.\left.q_{3}\right)\right) R_{33}\right]$

$$
q_{5}=\operatorname{atan}\left(\frac{s\left(q_{5}\right)}{c\left(q_{5}\right)}\right)
$$

$$
s\left(q_{6}\right)=\left[\left(c\left(q_{4}\right) \cdot s\left(q_{1}\right)+c\left(q_{1}\right) \cdot s\left(q_{2}+q_{3}\right) \cdot s\left(q_{4}\right)\right) R_{11}+\right.
$$
$\left(-c\left(q_{1}\right) \cdot c\left(q_{4}\right)+s\left(q_{1}\right) \cdot s\left(q_{2}+q_{3}\right) \cdot s\left(q_{4}\right)\right) R_{21}-\left(c\left(q_{2}+\right.\right.$ $\left.\left.\left.q_{3}\right) \cdot s\left(q_{4}\right)\right) R_{31}\right]$

$$
c\left(q_{6}\right)=\left[\left(c\left(q_{4}\right) \cdot s\left(q_{1}\right)+c\left(q_{1}\right) \cdot s\left(q_{2}+q_{3}\right) \cdot s\left(q_{4}\right)\right) R_{12}+\right.
$$

$\left(-c\left(q_{1}\right) \cdot c\left(q_{4}\right)+s\left(q_{1}\right) \cdot s\left(q_{2}+q_{3}\right) \cdot s\left(q_{4}\right)\right) R_{22}-\left(c\left(q_{2}+\right.\right.$

$\left.\left.q_{3}\right) \cdot s\left(q_{4}\right) R_{32}\right]$

$$
q_{6}=\operatorname{atan}\left(\frac{s\left(q_{6}\right)}{c\left(q_{6}\right)}\right)
$$

\section{RESULTS}

In order to verify its exact kinematics equation to control the end-effector, we analyzed the results using Matlab [9]. According to these coming from the inverse kinematics equation of the angles of joint, obtained the position of the end-effector.

Table (2): First experiment parameters

\begin{tabular}{|l|l|c|c|c|c|c|}
\hline \multirow{2}{*}{$\begin{array}{c}\text { Analyzing } \\
\text { Parameter }\end{array}$} & \multicolumn{7}{|c|}{ Manipulator Angles } \\
\cline { 2 - 7 } & $\boldsymbol{q}_{\mathbf{1}}$ & $\boldsymbol{q}_{\mathbf{2}}$ & $\boldsymbol{q}_{\mathbf{3}}$ & $\boldsymbol{q}_{\mathbf{4}}$ & $\boldsymbol{q}_{\mathbf{5}}$ & $\boldsymbol{q}_{\mathbf{6}}$ \\
\hline Initial Angle & 0 & 90 & -45 & 0 & 0 & 0 \\
\hline Final Angle & 45 & 45 & -90 & 45 & 45 & 45 \\
\hline Start Time & 1 & 101 & 201 & 301 & 401 & 600 \\
\hline Finish Time & 100 & 200 & 300 & 400 & 600 & 600 \\
\hline
\end{tabular}

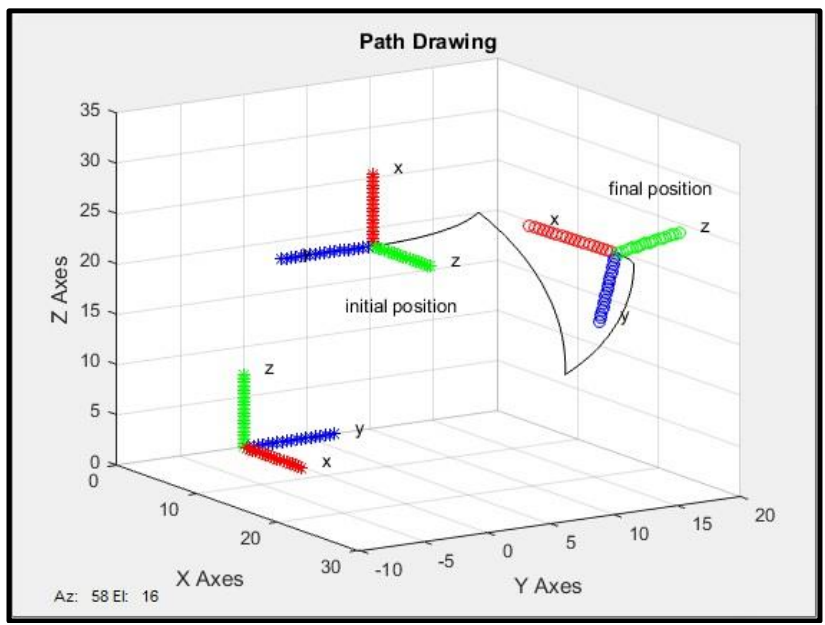

Figure (6): Path Drawing

Table (3): Second experiment parameters

\begin{tabular}{|l|l|c|c|c|c|c|}
\hline \multirow{2}{*}{$\begin{array}{c}\text { Analyzing } \\
\text { Parameter }\end{array}$} & \multicolumn{7}{|c|}{ Manipulator Angles } \\
\cline { 2 - 7 } & $\boldsymbol{q}_{\mathbf{1}}$ & $\boldsymbol{q}_{\mathbf{2}}$ & $\boldsymbol{q}_{\mathbf{3}}$ & $\boldsymbol{q}_{\mathbf{4}}$ & $\boldsymbol{q}_{\mathbf{5}}$ & $\boldsymbol{q}_{\mathbf{6}}$ \\
\hline Initial Angle & 0 & 90 & -45 & 0 & 0 & 0 \\
\hline Final Angle & 45 & 15 & -90 & 45 & 45 & 45 \\
\hline Start Time & 1 & 101 & 201 & 301 & 401 & 600 \\
\hline Finish Time & 100 & 200 & 300 & 400 & 600 & 600 \\
\hline
\end{tabular}




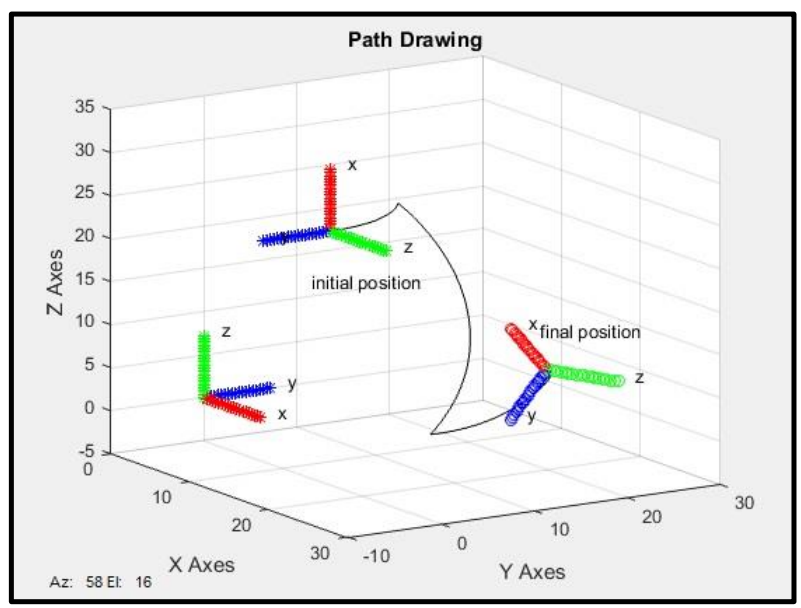

Figure (7): Path Drawing

The Total time for movement is $600 \mu S$, and angles measurement's unit is degree.

\section{CONCLUSION}

In this paper, the kinematics of the six-degree-of-freedom industrial manipulator prototype was analyzed. The proposed method can obtain the kinematics equation at the same time with the geometric structure and the homogeneous transformation matrix to control the manipulator. Finally, the paper presents the robot arm successfully utilizing Matlab Software to imitate the kinematics data and actual experiments. The experimental result verifies the validity of the kinematics equation.

\section{REFERENCES}

[1] S. Shrivastava, "Forward Kinematics of Articulated Robotic Arm," International Journal of Research and Scientific Innovation (IJRSI), vol. IV, no. VIII, pp. 7882, 2017.

[2] J. Iqbal, "Modeling and Analysis of a 6 DOF Robotic Arm," Canadian Journal on Electrical and Electronics Engineering, vol. 3, no. 6, pp. 300-306, 2012.

[3] Z. Hou, "Kinematics analysis and self - collision detection of Truss type multi-robot," in 52nd CIRP Conference on Manufacturing Systems, Shanghai, China, 2019.

[4] R. V. V. Petrescu, "Geometry and Inverse Kinematic at the MP3R Mobile Systems," Journal of Mechatronics and Robotics, vol. 1, no. 2, 2017.

[5] S. M. O. a. A. M. Djuric, "Fanuc Family Inverse Kinematics Modeling, Validation and," in $S A E$ International, California, 2016.

[6] M. G. Krishnan, "Kinematic Analysis and Validation of an Industrial," in IEEE TENCON Conference, Kerala, 2019.

[7] Z. S. Jianyu Wang, "Inverse Kinematics of 6-DoF Robot Manipulator via Analytic Solution," in International Conference on Robotics and Biomimetics, Kuala Lumpur, Malaysia, 2018.

[8] F. Liu, "Kinematic analysis and simulation of a 3-DOF robotic," in IEEE International Conference on "Computational Intelligence and Communication Technology", Ghaziabad, 2017.

[9] "MATLAB 2018a, The MathWorks, Inc., Natick, Massachusetts, United States.". 